H. Ito

Nagoya Math. J.

Vol. 96 (1984), $95-112$

\title{
CONGRUENCE RELATIONS OF ANKENY-ARTIN-CHOWLA TYPE FOR PURE CUBIC FIELDS
}

\author{
HIROSHI ITO
}

\section{§1. Introduction}

Ankeny, Artin and Chowla [1] proved a congruence relation among the class number, the fundamental unit of real quadratic fields, and the Bernoulli numbers. Our aim of this paper is to prove similar congruence relations for pure cubic fields. For this purpose, we use the Hurwitz numbers associated with the elliptic curve defined by $y^{2}=4 x^{3}-1$ instead of the Bernoulli numbers ( $\S 3)$. As a corollary to the main theorem ( $\S 5$ ), we have the following:

For a prime number $p$ congruent to -1 modulo 9 , let $h$ and $t+u \sqrt[3]{p}+v \sqrt[3]{p^{2}}>1$ be the class number and the fundamental unit of the pure cubic field $Q(\sqrt[3]{p})$ respectively, where $t, u$ and $v$ are rational numbers. Then we have:

$$
\begin{aligned}
2 u h & \equiv G_{\left(p^{2}-1\right) / 3} \bmod p, \\
2\left(2 v-u^{2}\right) h & \equiv G_{2\left(p^{2}-1\right) / 3} \bmod p .
\end{aligned}
$$

Here $G_{k}(k \geqq 2)$ are rational numbers defined by the power series expansion of the Weierstrass $p$-function satisfying $p^{\prime}(z)^{2}=4 p(z)^{3}-1$ :

$$
p(z)=\frac{1}{z^{2}}+\sum_{k=2}^{\infty}(k-1) G_{k} z^{k-2} .
$$

Let $m>0$ be a cube-free rational integer which has a prime divisor $p \neq 2,3$, and $p$ a prime ideal of $K=Q(\sqrt{-3})$ over $p$. In this paper, we shall prove similar congruence relations modulo $\mathfrak{p}$ for the pure cubic field $\boldsymbol{Q}(\sqrt[3]{m})$. For this purpose, we first translate, in Section 2, the analytic class number formula into the form

$$
\text { (the fundamental unit })^{h}=(\text { the elliptic unit), }
$$

Received October 7, 1983. 
and then, following the idea of Robert [11], we take Kummer's logarithmic derivatives of both sides. In the final section, we shall give some discussion concerning the $p$-adic $L$-functions of Lichtenbaum [6].

Throughout, we denote by $\overline{\boldsymbol{Q}}$ the algebraic closure of the rational number field $\boldsymbol{Q}, \boldsymbol{C}$ the complex number field; and $\boldsymbol{C}_{p}$ the completion of the algebraic closure of the $p$-adic number field $\boldsymbol{Q}_{p}$. We fix an embedding $i_{\infty}$ of $\overline{\boldsymbol{Q}}$ into $\boldsymbol{C}$ and an embedding $i_{p}$ of $\overline{\boldsymbol{Q}}$ into $\boldsymbol{C}_{p}$ such that $i_{p}(\mathfrak{p})$ is contained in the valuation ideal of $C_{p}$. Via these embeddings, the algebraic numbers in $C$ will be identified with the algebraic numbers in $C_{p}$. Denote by $h$ and $\varepsilon>1$ the class number and the fundamental unit of the pure cubic field $\boldsymbol{Q}(\delta)$ respectively. Here $\delta$ is the real cube root of $m$.

\section{§2. The analytic class number formula}

In this section, we translate the analytic class number formula for $\boldsymbol{Q}(\delta)$ into the form which is suitable for the later applications ((2), (7)). Until the end of Section 3, the discussion will take place inside $C$. Put $H=$ $K(\delta)$ and denote by $\mathcal{O}_{K}$ the ring of integers of $K$. Note that $m$ is uniquely expressed as $m=a b^{2}$, where $a$ and $b$ are positive integers which are square-free and prime to each other. Then the conductor of the abelian extension $H / K$ is given by the ideal $(f)=f \mathcal{O}_{K}$. Here $f$ is the rational integer defined as follows (cf. Hasse [4] and LeVeque [5]):

$$
f=\left\{\begin{aligned}
a b & \text { if } a^{2} \equiv b^{2} \bmod 9, \\
3 a b & \text { otherwise } .
\end{aligned}\right.
$$

The ray class group $\mathrm{Cl}(f)$ of $K$ modulo $(f)$ is naturally isomorphic to $\left(\mathcal{O}_{K} / f \mathcal{O}_{K}\right)^{\times} / \bar{\mu}$, where $\bar{\mu}$ is the image of the group $\mu$ of units of $K$ in $\left(\mathcal{O}_{K} / f \mathcal{O}_{K}\right)^{\times}$. By the assumption on $m, f$ has a prime divisor $p \neq 2,3$, so that $\bar{\mu}$ has order 6. If $\alpha \in \mathcal{O}_{K}$ is prime to $f$, we denote by $C_{\alpha}$ the element of $\mathrm{Cl}(f)$ represented by $(\alpha)$.

Denote by $(\alpha / \beta)_{3}$ the cubic residue symbol in $K$ and put $\chi=(m / \cdot)_{3}$. Then the map $C_{\alpha} \mapsto \chi(\alpha)$ is well-defined and gives a character of $\mathrm{Cl}(f)$ corresponding to $H / K$. We denote this character also by $\chi$. For the Dedekind zeta function $\zeta_{\boldsymbol{Q}(\delta)}(s)$ of $\boldsymbol{Q}(\delta)$, we see

$$
\zeta_{Q(\delta)}(s)=\zeta(s) L_{K}(s, \chi)
$$

from Meyer [8]. It follows from the analytic class number formula that

$$
h \log \varepsilon=L_{K}^{\prime}(0, \varepsilon) \text {. }
$$


Let $H_{f}$ be the ray class field of $K$ modulo $(f)$. Take and fix $\gamma \in \mathcal{O}_{K}$ such that $(\gamma, 6 f)=1$ and $\chi(\gamma) \neq 1$. If we use the ray class invariant $\varphi_{f}(C)$ modulo $(f)$ defined in Section 2 of Robert [10], we see

$$
\begin{aligned}
L_{K}^{\prime}(0, \chi) & =-\frac{1}{12 f} \sum_{C \in \mathrm{Cl}(f)} \chi(C) \log \left|\varphi_{f}(C)\right|^{2} \\
& =\frac{1}{12 f} \log \left|N_{H f / H}\left(\varphi_{f}\left(C_{\gamma}\right) / \varphi_{f}\left(C_{1}\right)\right)\right|^{2} .
\end{aligned}
$$

Hence we obtain

$$
\varepsilon^{12 f h}=\left|N_{H f / H}\left(\varphi_{f}\left(C_{\gamma}\right) / \varphi_{f}\left(C_{1}\right)\right)\right|^{2} .
$$

Since $\overline{N_{H_{f} / H}\left(\varphi_{f}\left(C_{\gamma}\right)\right)}=N_{H_{f} / H}\left(\varphi_{f}\left(C_{\gamma}^{-1}\right)\right)$, we also have

$$
\varepsilon^{12 f h}=\prod_{C \in \mathbb{C}(f)} \varphi_{f}(C)^{-(\chi(C)+\chi(C)-1)} .
$$

Now we consider the $f$-th root $(>0)$ of the right hand side of (2). Our technique here is borrowed from [10]. Let $p(z)$ be the Weierstrass $p$-function which satisfies

$$
p^{\prime}(z)^{2}=4 p(z)^{3}-1
$$

Denote by $L$ the period lattice of $p(z)$. We may write $L=\mathcal{O}_{K} \Omega$ with $\Omega$ real and positive. For $\alpha \in \mathcal{O}_{K}$, denote by $\alpha^{\prime}$ the conjugate of $\alpha$ and put $N \alpha=\alpha \alpha^{\prime}$. Let $\sigma(z)$ be the Weierstrass $\sigma$-function of $L$, and put

$$
\begin{aligned}
& \theta(z)=\Delta(L) \sigma^{12}(z), \\
& \phi(z ; \alpha)=\theta(\alpha z) / \theta(z)^{N \alpha} \quad\left(\alpha \in \mathcal{O}_{K}\right) .
\end{aligned}
$$

Here $L(L)$ is the discriminant of $L$ which is equal to -27 . It should be remarked that $\phi(z ; \alpha)$ is an elliptic function with respect to $L$. More precisely, we have

$$
\phi(z ; \alpha)=\alpha^{12} \Delta(L)^{1-N \alpha} \prod_{\substack{\alpha \beta=0 \\ \beta \neq 0}}(p(z)-p(\beta))^{6}
$$

where the product is taken over the non-zero $\alpha$-division points $\beta$ of $C / L$ (Corollary 2.6 of [6]).

Because the number of roots of unity in $H$ is equal to that of $K$, by Lemma 6 of [10], we can take $\beta_{j} \in \mathcal{O}_{K}$ and $m_{j} \in Z(j \in J)$ such that

$$
\left\{\begin{array}{l}
N \gamma-1+\sum_{j \in J} m_{j}\left(N \beta_{j}-1\right)=0, \\
\chi\left(C_{\beta_{j}}\right)=1, \quad\left(\beta_{j}, 6 f\right)=1 \quad(j \in J) .
\end{array}\right.
$$


Here $J$ is a finite index set. We fix $\left\{\beta_{j}\right\}_{j \in J}$ and $\left\{m_{j}\right\}_{j \in J}$ which satisfy (5) throughout this paper. Set $\tau=f^{-1} \Omega$, and put

$$
\eta=\phi(\tau ; \gamma) \prod_{j \in J} \phi\left(\tau ; \beta_{j}\right)^{m_{j}} \text {. }
$$

LEMMA 1. (i) $\eta \in H_{f}$.

(ii) $N_{H f_{f} / H}(\eta)^{f}=N_{H f / H}\left(\varphi_{f}\left(C_{\gamma}\right) / \varphi_{f}\left(C_{1}\right)\right)$.

Proof. It is seen from (4) that $\phi(z ; \alpha)$ is a polynomial of $p(z)$ with coefficients in $K$ and $\phi(\zeta z ; \alpha)=\phi(z ; \alpha)$ for all $\zeta \in \mu$. Therefore $\phi(\tau ; \alpha) \in H_{f}$ for any $\alpha \in \mathcal{O}_{K}$, from which follows (i). To prove (ii), we note that

$$
\phi(\tau ; \alpha)^{f}=\varphi_{f}\left(C_{\alpha}\right) / \varphi_{f}\left(C_{1}\right)^{N \alpha}
$$

if $(\alpha, f)=1$, and that

$$
N_{H \mathcal{f} / H}\left(\varphi_{f}\left(C_{\beta_{j}}\right)\right)=N_{H_{\mathcal{f}} / H}\left(\varphi_{f}\left(C_{1}\right)\right)
$$

for all $j \in J$ (cf. $\S 2$ and $\S 10$ of [6]). Then, from (5), we see

$$
\begin{aligned}
N_{H_{f} / H}(\eta)^{f} & =N_{H_{f} / H}\left[\varphi_{f}\left(C_{\gamma}\right) \varphi_{f}\left(C_{1}\right)^{-N_{\gamma}+{ }_{j \in J} m_{j}\left(1-N \beta_{j}\right)}\right] \\
& =N_{H_{f} / H}\left(\varphi_{f}\left(C_{\gamma}\right) / \varphi_{f}\left(C_{1}\right)\right),
\end{aligned}
$$

which completes the proof.

Since $\varepsilon>0$ and $N_{H_{f} / H}(\eta \bar{\eta})=\left|N_{H_{f} / H}(\eta)\right|^{2}>0$, we obtain

$$
\varepsilon^{12 h}=N_{H_{f} / H}(\eta \bar{\eta})
$$

from (2). Note that

$$
\bar{\eta}=\phi\left(\tau ; \gamma^{\prime}\right) \prod_{j \in J} \phi\left(\tau ; \beta_{j}^{\prime}\right)^{m_{j}}
$$

\section{§3. The generalized Hurwitz numbers}

We first summarize some notation and facts concerning the elliptic curve $E$ defined by the equation

$$
y^{2}=4 x^{3}-1 .
$$

The map $z \mapsto \xi(z)=\left(p(z), p^{\prime}(z)\right)$ gives an isomorphism from $C / L$ onto the complex points of $E$. As usual, we identify $\mathcal{O}_{K}$ with the endomorphism ring of $E$ in such a way that the endomorphism corresponding to $\alpha \in \mathcal{O}_{K}$ is given by $\xi(z) \mapsto \xi(\alpha z)$. For $\alpha \in \mathcal{O}_{K}$, we denote by $F_{\alpha}$ the field obtained by adjoining to $K$ the coordinates of $\alpha$-division points of $E$. It is known that $F_{\alpha} / K$ is abelian and every prime ideal of $K$ which ramifies in $F_{\alpha}$ is a 
divisor of $6 \alpha$. For $\alpha \in \mathcal{O}_{K},(\alpha, 6)=1$, denote by $\alpha^{*}$ the generator of the ideal $(\alpha)$ such that

$$
\alpha^{*} \equiv\left(\frac{-1}{\alpha}\right)_{2} \bmod 3
$$

where $(-1 / \alpha)_{2}$ is the quadratic residue symbol in $K$. Then the next lemma follows from the results of Davenport and Hasse [3].

LEMMA 2. Let $\nu, \mu \in \mathcal{O}_{K},(\nu, 6 \mu)=1$, and let $Q_{\mu}$ be a $\mu$-division point of $E$. Then

$$
\boldsymbol{Q}_{\mu}^{\sigma_{\nu}}=\nu^{*} \boldsymbol{Q}_{\mu},
$$

where $\sigma_{\nu}$ is the Artin automorphism of the ideal ( $\nu$ ) with respect to $F_{\mu} / K$.

Let $\pi$ be the generator of $p$ such that $\pi^{*}=\pi$ and set $q=N \pi$. Define $f_{0}, m_{0} \in \mathcal{O}_{K}$ by $f=\pi f_{0}, m=\pi m_{0}$. It is seen from (1) that $\left(\pi, f_{0}\right)=1$. Hence there exist $\tau_{1}, \tau_{2} \in C$, which are uniquely determined modulo $L$, such that

$$
\tau \equiv \tau_{1}+\tau_{2}, \quad \pi \tau_{1} \equiv f_{0} \tau_{2} \equiv 0 \quad \bmod L
$$

Here $\tau=f^{-1} \Omega$ as in Section 2. Define the points $P, P_{1}, P_{2}$ of $E$ by

$$
P=\xi(\tau), \quad P_{i}=\xi\left(\tau_{i}\right) \quad(i=1,2) .
$$

Let $\mathfrak{n}$ be an integral ideal of $K$. We call a function $\lambda: \mathcal{O}_{k} \rightarrow \overline{\boldsymbol{Q}}$ a Dirichlet character defined modulo $n$ if there exists a character $\tilde{\lambda}$ of $\left(\mathcal{O}_{K} / \mathfrak{n}\right)^{\times}$ such that $\lambda(\alpha)=\tilde{\lambda}(\alpha \bmod \mathfrak{n})$ for $(\alpha, \mathfrak{n})=1$, and $\lambda(\alpha)=0$ otherwise. We can define the conductor of $\lambda$ by the usual way. A Dirichlet character is called primitive if it is defined modulo its conductor. In the following, all Dirichlet characters we consider will be primitive. Write $m=a b^{2}$ as explained in Section 2. We can assume $p \mid a$ without loss of generality by replacing $m$ by $m^{2} / b^{3}$ if necessary. Then a Dirichlet character $\chi_{2}$ modulo $\left(f_{0}\right)$ is defined by

$$
\chi(\alpha)=\chi_{1}(\alpha) \chi_{2}(\alpha), \quad \chi_{1}(\alpha)=\left(\frac{\alpha}{\pi}\right)_{3}
$$

for $\alpha \in \mathcal{O}_{K},(\alpha, f)=1$. We also view $\chi$ and $\chi_{1}$ as Dirichlet characters defined modulo $(f)$ and $(\pi)$ respectively.

Denote by $\zeta(z)$ the Weierstrass $\zeta$-function of $L$, i.e., $\zeta(z)=(d / d z) \log \sigma(z)$. For any $\ell \in L$ there is a constant $\kappa(\ell)$ such that

$$
\zeta(z+\ell)=\zeta(z)+\kappa(\ell) .
$$


The function $\ell \mapsto \kappa(\ell)$ is clearly linear in $\ell$, and we extend it by $R$-linearity to a function on $C$. Let $w \in C / L$ and take a representative $r_{w} \in C$ of $w$. Then $\zeta\left(z+r_{w}\right)-\kappa\left(r_{w}\right)$ does not depend on the choice of $r_{w}$. We put $\zeta^{*}(z ; w)=\zeta\left(z+r_{w}\right)-\kappa\left(r_{w}\right)$ (cf. Lemma 3.1 of [6]). For $\lambda=\chi_{2}$ or $\chi_{2}^{-1}$, we define the generalized Hurwitz numbers $G_{k, \lambda}(k \geqq 0)$, following [6], by

$$
\sum_{\alpha \in\left(O_{K} / f_{0} O_{K}\right)^{x}} \lambda(\alpha)^{-1} \zeta^{*}\left(z ; \alpha \tau_{2}\right)=-\sum_{k=0}^{\infty} G_{k, \lambda} z^{k-1} .
$$

It is easily seen that

$$
G_{0,2}=\left\{\begin{aligned}
-1 & \text { if }\left(f_{0}\right)=(1), \\
0 & \text { otherwise } .
\end{aligned}\right.
$$

As is shown in Section 7 of [6], $G_{k, 2}(k \geqq 1)$ are numbers related to Hecke $L$-functions associated with $K$. Because $-(d / d z) \zeta(z)=p(z)$, we have $G_{k, \lambda}=G_{k}(k \geqq 2)$ if $\left(f_{0}\right)=(1)$, where $G_{k}$ are the numbers defined in the introduction.

LEMMA 3. (i) $G_{k, \chi_{2}}, G_{k, x_{2}^{-1}} \in F_{f_{0}}(k \geqq 0)$.

(ii) $G_{k, x_{2}} / \sqrt[3]{m_{0}}, G_{k, x_{2}^{-1}} / \sqrt[3]{m_{0}^{2}} \in K(k \geqq 0)$.

(iii) When $q=p^{2}$, we have

$$
G_{k, x_{2}} / \sqrt[3]{m_{0}}, \quad G_{k, x_{2}^{-1}} / \sqrt[3]{m_{0}^{2}} \in \boldsymbol{Q} \quad(k \geqq 0)
$$

if $\sqrt[3]{m_{0}}$ is real.

Proof. We only consider the assertions concerning to the numbers $G_{k, \chi_{2}}$, because those concerning to $G_{k, x_{2}^{-1}}$ can be proved similarly. If $\left(f_{0}\right)=(1)$, them $m_{0}=-1, G_{0, \chi_{2}}=-1, G_{1, \chi_{2}}=0$, and $G_{k, \chi_{2}}=G_{k}(k \geqq 2)$, from which all the assertions follow. Assume $\left(f_{0}\right) \neq(1)$. Because $\chi_{2}(-1)$ $=1$, we have

$$
\frac{1}{2} \sum_{\substack{\alpha \bmod f_{0} \\\left(\alpha, f_{0}\right)=1}} \chi_{2}(\alpha)^{-1} \frac{p^{\prime}(z)}{p(z)-p\left(\alpha \tau_{2}\right)}=-\sum_{k=1}^{\infty} G_{k, \chi_{2}} z^{k-1}
$$

by Lemma 3.3 of [6]. Then the assertion (i) is clear from the definition of $F_{f_{0}}$.

To prove (ii), let ( $\nu$ ) be an integral ideal of $K$ prime to $6 f$, and $\sigma_{\nu}$ the Artin automorphism of $(\nu)$ with respect to $F_{f_{0}}\left(\sqrt[3]{m_{0}}\right) / K$. By Lemma 2,

$$
-\sum_{k=1}^{\infty} G_{k, x_{2}}^{\sigma_{\nu}} z^{k-1}=\frac{1}{2} \sum_{\alpha} \chi_{2}(\alpha)^{-1} \frac{p^{\prime}(z)}{p(z)-p\left(\alpha \tau_{2}\right)^{\sigma_{\nu}}}
$$




$$
\begin{aligned}
& =\frac{1}{2} \sum_{\alpha} \chi_{2}(\alpha)^{-1} \frac{p^{\prime}(z)}{p(z)-p\left(\nu^{*} \alpha \tau_{2}\right)} \\
& =\frac{1}{2} \chi_{2}\left(\nu^{*}\right) \sum_{\alpha} \chi_{2}(\alpha)^{-1} \frac{p^{\prime}(z)}{p(z)-p\left(\alpha \tau_{2}\right)} \\
& =-\chi_{2}\left(\nu^{*}\right) \sum_{k=1}^{\infty} G_{k, x_{2}} z^{k-1}
\end{aligned}
$$

Hence

$$
G_{k, \chi_{2}}^{\sigma_{\nu}-1}=\chi_{2}\left(\nu^{*}\right)=\left(\frac{m}{\nu}\right)_{3}\left(\frac{\nu^{*}}{\pi}\right)_{3}^{-1}=\left(\frac{m}{\nu}\right)_{3}\left(\frac{\pi}{\nu}\right)_{3}^{-1}=\left(\frac{m_{0}}{\nu}\right)_{3} .
$$

On the other hand,

$$
\sqrt[3]{m_{0}^{\sigma_{\nu}-1}}=\left(\frac{m_{0}}{\nu}\right)_{3}
$$

Thus (ii) is proved.

Finally assume $q=p^{2}$. Then it is easily seen that $G_{k, \chi_{2}}$ are real. This proves (iii).

\section{§4. Kummer's logarithmic derivatives}

In this section, we introduce certain group homomorphisms $\psi_{k}$ $(1 \leqq k \leqq q-1)$ which are used in [11] and were referred to as Kummer's logarithmic derivatives in the introduction (See also $\S 3$ and $\S 4$ of Coates and Wiles [2].). Let $M_{0}$ be a finite abelian extension of $K$ such that the prime ideal $\mathfrak{p}$ does not ramify at $M_{0} / K$, and put $M=M_{0} L$, where $L=F_{\pi}$. Then, since $L / K$ is an abelian extension of degree $q-1$ where $p$ ramifies completely, the prime ideal $\mathfrak{q}$ of $M_{0}$ corresponding to the fixed embedding $i_{p}: \overline{\boldsymbol{Q}} \hookrightarrow C_{p}$ ramifies completely at $M / M_{0}$ and $\left[M: M_{0}\right]=q-1$. Denote by $\mathfrak{Q}$ the prime ideal of $M$ above q. Let $M_{\mathfrak{Q}}$ and $M_{0, q}$ be the completions of $M$ and $M_{0}$ at $\mathfrak{Q}$ and $q$ respectively. For any subfield $N$ of $C_{p}$, denote by $\mathcal{O}(N)$ the ring of integers of $N, \mathfrak{m}(N)$ the maximal ideal of $\mathcal{O}(N)$. Put $\mathcal{O}_{\mathfrak{a}}=\mathcal{O}\left(M_{\mathfrak{0}}\right), \mathfrak{m}_{\mathfrak{a}}=\mathfrak{m}\left(M_{\mathfrak{o}}\right), \mathcal{O}_{\mathfrak{q}}=\mathcal{O}\left(M_{0, q}\right)$, and $\mathfrak{m}_{\mathfrak{q}}=\mathfrak{m}\left(M_{0, q}\right)$. We remark here that, in the later sections, we shall apply the argument of this section to $M_{0}=F_{f_{0}}$.

For any prime element $\Lambda$ of $M_{\mathfrak{Q}}$ we can define group homomorphisms

$$
\psi_{k}: M_{\mathscr{Q}}^{\times} \longrightarrow \mathcal{O}_{a} / \mathfrak{m}_{a} \quad(1 \leqq k \leqq q-1)
$$

as follows. First, suppose $u$ is a unit of $M_{\mathfrak{Q}}$ congruent to 1 modulo $\mathfrak{m}_{\mathfrak{Q}}$. Choose a power series $f(T)=1+\sum_{k=1}^{\infty} a_{k} T^{k}$, with coefficients in $\mathcal{O}_{q}$, such 
that $u=f(\Lambda)$. For $1 \leqq k \leqq q-1$, we define $\psi_{k}(u)$ to be the residue class in $\mathcal{O}_{\mathfrak{q}} / \mathfrak{m}_{\mathrm{q}}$ of the coefficient of $T^{k}$ in $T(d / d T) \log f(T)$. Since $M_{\mathfrak{a}} / M_{0, \mathrm{q}}$ is completely ramified, $\psi_{k}(u)$ is independent of the choice of $f(T)$. Because any element $\alpha$ of $M_{\Omega}$ is written uniquely in the form $\Lambda^{n} \zeta u\left(n \in Z, \zeta^{N Q-1}=1\right.$, $\left.u \equiv 1 \bmod \mathfrak{m}_{\mathfrak{a}}\right)$, we can extend $\psi_{k}$ on $M_{\mathfrak{a}}$ by defining

$$
\psi_{k}(\Lambda)=\psi_{k}(\zeta)=0 \text {. }
$$

The homomorphisms $\psi_{k}$ depend on the choice of the prime element $\Lambda$. We shall now make a particular choice of $\Lambda$. Let $\mathfrak{P}$ be the prime ideal of $L$ over $\mathfrak{p}$, and let $L_{\mathfrak{\beta}}$ denote the completion of $L$ at $\mathfrak{P}$ and $K_{\mathfrak{p}}$ the completion of $K$ at $\mathfrak{p}$. Set $\mathcal{O}_{\mathfrak{p}}=\mathcal{O}\left(K_{\mathfrak{p}}\right)$ and $\mathfrak{m}_{\mathfrak{p}}=\mathfrak{m}\left(K_{\mathfrak{p}}\right)$. Let $E$ be the elliptic curve defined by (8), and let $\hat{E}$ denote the formal group over $\mathcal{O}_{p}$ of the kernel of reduction modulo $\mathfrak{m}_{\mathrm{p}}$ on $E$, with parameter $t=-2 x / y$ (Tate [12]). By the definition of $\pi$, the endomorphism $\pi$ on $E$ reduces to the Frobenius endomorphism of $E$ modulo $\mathfrak{p}$. Therefore $\hat{E}$ is a Lubin-Tate formal group for the uniformizing parameter $\pi$ of $K_{p}$ (Lubin and Tate [7]), and is isomorphic over $\mathcal{O}_{p}$ to the formal group $\mathscr{E}$ defined by the endomorphism

$$
[\pi]_{\delta}(T)=\pi T+T^{q} .
$$

Denote by $w$ the isomorphism from $\hat{E}$ to $\mathscr{E}$ over $\mathcal{O}_{\mathfrak{p}}$, and put $\Lambda=w\left(t\left(P_{1}\right)\right)$. Then $t\left(P_{1}\right)$ and $\Lambda$ are prime elements of $L_{\mathfrak{p}}$. Since $M_{\mathfrak{Q}} / L_{\mathfrak{p}}$ is unramified, they are also prime elements of $M_{\mathfrak{Q}}$. In the following, we consider the homomorphisms $\psi_{k}(1 \leqq k \leqq q-1)$ with respect to this $\Lambda$. It is seen from $[\pi]_{\delta}(\Lambda)=0$ that

$$
\Lambda^{q-1}=-\pi
$$

Although $\Lambda$ depends on the choice of the embedding $i_{p}, \Lambda^{(q-1) / 3}$ gives a cube root of $-\pi$ which is independent of $i_{p}{ }^{1)}$ In fact, $\Lambda^{(q-1) / 3}$ is contained in $L$ and is determined by

$$
\Lambda^{(q-1) / 3} \equiv t\left(P_{1}\right)^{(q-1) / 3} \bmod \mathfrak{P}^{(q-1) / 3+1} .
$$

From this congruence, it is also seen that $\Lambda^{(q-1) / 3}$ is the real cube root of $-\pi=p$ in case $q=p^{2}$.

The homomorphisms $\psi_{k}$ have the following property which will be

1) Concerning this point, the author is indebted to Masato Kamei for pointing out an error in the original manuscript. 
used later. If we identify $\operatorname{Gal}\left(M_{\mathfrak{O}} / M_{0, q}\right)$ with $\operatorname{Gal}\left(L_{\mathfrak{P}} / K_{\mathrm{p}}\right)$, and with $\operatorname{Gal}(L / K)$ naturally, we obtain the isomorphism

$$
\left(\mathcal{O}_{K} / \mathfrak{p}\right)^{\times} \longrightarrow \operatorname{Gal}\left(M_{\mathfrak{2},} / M_{0, \mathfrak{q}}\right)
$$

by considering the actions of both groups on the group of $\mathfrak{p}$-division points of $E$. Denote by $g_{\nu}$ the element of $\operatorname{Gal}\left(M_{\mathfrak{0}} / M_{0, q}\right)$ corresponding to $\nu$ modulo $\mathfrak{p} \in\left(\mathcal{O}_{K} / \mathfrak{p}\right)^{\times}$. The following lemma is proved by the same way as in Proposition 45 of [11].

LEMMA 4. Let $k$ be an integer such that $1 \leqq k \leqq q-1$. For any $\alpha \in M_{\mathfrak{a}}$ and $\nu \in \mathcal{O}_{K},(\nu, \pi)=1$, we have

$$
\psi_{k}\left(\alpha^{g_{\nu}}\right)=\nu^{k} \psi_{k}(\alpha)
$$

\section{§5. Main theorem}

We define $\sqrt[3]{\pi}$ and $\sqrt[3]{m_{0}}$ by

$$
\sqrt[3]{\pi}=-\Lambda^{(q-1) / 3}, \quad \delta=\sqrt[3]{\pi} \sqrt[3]{m_{0}} .
$$

The generalized Hurwitz numbers $G_{k, \chi_{2}}, G_{k, \chi_{2}^{-1}}$ and the cube root $\sqrt[3]{m_{0}}$ of $m_{0}$ defined above are elements of $F_{f_{0}}$. Although these numbers depend on the choice of the embedding $i_{\infty}: \overline{\boldsymbol{Q}} \longrightarrow C$, the numbers $G_{k, x_{2}} / \sqrt[3]{m_{0}}$ and $G_{k, \chi_{2}^{-1}} / \sqrt[3]{m_{0}^{2}}(k \geqq 0)$ are elements of $K$ which are independent of $i_{\infty}$ and $i_{p}$. Moreover they are rational numbers in case $q=p^{2}$ (cf. Lemma 3).

We are now ready to state the main theorem of this paper.

THEOREM. Let $m>0$ be a cube-free rational integer which is divisible by a prime number $p \neq 2,3$ and not divisible by $p^{2}$. Let $\mathfrak{p}$ be a prime ideal of $\boldsymbol{Q}(\sqrt{-3})$ over $p$ and $\pi$ its generator such that $\pi \equiv(-1 / \mathfrak{p})_{2} \bmod 3$. Define the Dirichlet character $\chi_{2}$ of $\boldsymbol{Q}(\sqrt{-3})$ by $(\mathrm{m} / \cdot)_{3}=(\cdot / \mathfrak{p})_{3} \chi_{2}$ and let $G_{k, \lambda}\left(\lambda=\chi_{2}, \chi_{2}^{-1}\right)$ be the Hurwitz numbers defined in Section 3. Further let $\sqrt[3]{m_{0}}$ be the cube root of $m_{0}=m / \pi$ defined as above. Then, if we denote by $h$ and $\varepsilon=t+u \sqrt[3]{m}+v \sqrt[3]{m^{2}}>1(t, u, v \in \boldsymbol{Q})$ the class number and the fundamental unit of the pure cubic field $\boldsymbol{Q}(\sqrt[3]{m})$ respectively, we have:

$$
\begin{aligned}
-2 \frac{u}{t} h & \equiv G_{\left(N_{\mathfrak{p}-1) / 3, x_{2}}\right.} \sqrt[3]{m_{0}} \bmod \mathfrak{p} \\
2\left(2 \frac{v}{t}-\left(\frac{u}{t}\right)^{2}\right) h & \equiv G_{2(N \mathfrak{p}-1) / 3, x_{2}^{-1}} / \sqrt[3]{m_{0}^{2}} \bmod \mathfrak{p} .
\end{aligned}
$$

Moreover, in case $p$ is congruent to -1 modulo 3 , both sides of the above 
congruences are rational numbers, and 'mod $\mathfrak{p}$ ' can be replaced by 'mod $p$ '. In this case, we also have $t \equiv 1 \bmod p$.

Remark. Suppose $m=p \equiv-1 \bmod 9$. Then we have $\pi=-p, m_{0}$ $=-1$, and $\sqrt[3]{m_{0}}=-1$. We also have $\left(f_{0}\right)=(1)$, hence $G_{k, x_{2}}=G_{k, x_{2}^{-1}}=G_{k}$ $(k \geqq 2)$. Therefore the statement in the introduction follows from the above theorem.

To prove the theorem we prepare a proposition. In the following, we set $M_{0}=F_{f_{0}}$ and use the notation of the previous section. In particular $M=F_{f} . \quad$ As is noted in the proof of Lemma $1, \phi(z ; \alpha)\left(\alpha \in \mathcal{O}_{K}\right)$ is a polynomial of $p(z)$ with coefficients in $K$ and $\phi(\zeta z ; \alpha)=\phi(z ; \alpha)$ for all $\zeta \in \mu$. It follows that $\phi\left(\tau_{1}+\mu \tau_{2} ; \alpha\right) \in M$ for any $\alpha, \mu \in \mathcal{O}_{K}$.

Proposition. Let $\alpha \in \mathcal{O}_{K},(\alpha, f)=1$, and let $k$ be an integer such that $1 \leqq k<q-1$. If $\lambda$ coinsides with $\chi_{2}$ or $\chi_{2}^{-1}$, we have

$$
\sum_{\substack{\mu \bmod f_{0} \\\left(\mu, f_{0}\right)=1}} \lambda(\mu)^{-1} \psi_{r_{k}}\left(\phi\left(\tau_{1}+\mu \tau_{2} ; \alpha\right)\right)=12\left(N \alpha-\alpha^{k} \lambda(\alpha)\right) G_{k, \lambda} \bmod \mathrm{m}_{\mathrm{q}} .
$$

Proof. Our proof is almost the same as that of Coates and Wiles [2] or Robert [11]. For simplicity, we assume $\left(f_{0}\right) \neq(1)$. The case $\left(f_{0}\right)=(1)$ is treated in [11], Proposition 46. Put $\phi(z)=\phi(z ; \alpha)$. We first note that

$$
\frac{d}{d z} \log \theta(z)=12 \zeta(z) \text {. }
$$

For $\mu \in \mathcal{O}_{K},\left(\mu, f_{0}\right)=1$, we define the complex numbers $d_{k}(\mu)(k \geqq 0)$ by

$$
\zeta^{*}\left(z ; \mu \tau_{2}\right)=\sum_{k=0}^{\infty} d_{k}(\mu) z^{k-1}
$$

Then, from the definition of $G_{k, \lambda}$, it is seen that

$$
G_{k, \lambda}=-\sum_{\substack{\mu \bmod f_{0} \\\left(\mu, f_{0}\right)=1}} \lambda(\mu)^{-1} d_{k}(\mu) \text {. }
$$

Hence

$$
\sum_{\mu} \lambda(\mu)^{-1}\left[\alpha^{k} d_{k}(\alpha \mu)-(N \alpha) d_{k}(\mu)\right]=\left(N \alpha-\alpha^{k} \lambda(\alpha)\right) G_{k, \lambda}
$$

On the other hand, since $\phi(z)=\theta(\alpha z) / \theta(z)^{N \alpha}$, we have

$$
\begin{aligned}
z \frac{d}{d z} \log \phi\left(z+\mu \tau_{2}\right) & =12 \alpha z \zeta\left(\alpha z+\alpha \mu \tau_{2}\right)-12(N \alpha) z \zeta\left(z+\mu \tau_{2}\right) \\
& =12 \sum_{k=0}^{\infty}\left[\alpha^{k} d_{k}(\alpha \mu)-(N \alpha) d_{k}(\mu)\right] z^{k} .
\end{aligned}
$$


It follows from the above remark on the function $\phi(z)$ that $\alpha^{k} d_{k}(\alpha \mu)$ - $(N \alpha) d_{k}(\mu)(k \geqq 0)$ are elements of $M_{0}$. We shall prove that these numbers are contained in $\mathcal{O}_{\mathfrak{q}}$ and

$$
\psi_{k}\left(\phi\left(\tau_{1}+\mu \tau_{2}\right)\right)=12\left[\alpha^{k} d_{k}(\alpha \mu)-(N \alpha) d_{k}(\mu)\right] \bmod \mathfrak{m}_{\mathfrak{q}}
$$

if $1 \leqq k<q-1$. Then the proof will be completed by (17).

Fix an integer $\mu \in \mathcal{O}_{K}$ such that $\left(\mu, f_{0}\right)=1$. The formula (4) and the addition theorem for $p(z)$ give

$$
\begin{aligned}
& \phi\left(z+\mu \tau_{2}\right) \\
& \quad=\alpha^{12} \Delta(L)^{1-N \alpha} \prod_{\substack{\alpha \beta=0 \\
\beta \neq 0}}\left(p\left(z+\mu \tau_{2}\right)-p(\beta)\right)^{6} \\
& \quad=\alpha^{12} \Delta(L)^{1-N \alpha} \prod_{\beta}\left[-p(z)-p\left(\mu \tau_{2}\right)+\frac{1}{4}\left(\frac{p^{\prime}(z)-p^{\prime}\left(\mu \tau_{2}\right)}{p(z)-p\left(\mu \tau_{2}\right)}\right)^{2}-p(\beta)\right]^{6} .
\end{aligned}
$$

Let $\ell$ be the isomorphism over $K_{\mathfrak{p}}$ from $\hat{E}$ to the formal additive group $G_{a}\left(G_{a}(X, Y)=X+Y\right)$, and $p(\ell(T))$ and $p^{\prime}(\ell(T))$ the formal power series obtained by substituting $z=\ell(T)$ in the Laurent expansions at the origin of $p(z)$ and $p^{\prime}(z)$ respectively. Then there exists a power series $a(T) \in$ $Z[[T]]$ such that $a(T) \equiv 1 \bmod$ degree 1 and

$$
p(\ell(T))=T^{-2} a(T), \quad p^{\prime}(\ell(T))=-2 T^{-3} a(T) .
$$

Moreover $x\left(P_{1}\right)=t\left(P_{1}\right)^{-2} a\left(t\left(P_{1}\right)\right)$ and $y\left(P_{1}\right)=-2 t\left(P_{1}\right)^{-3} a\left(t\left(P_{1}\right)\right)$ in $L_{\Re}$ (cf. [12]). Here $x\left(P_{1}\right)$ and $y\left(P_{1}\right)$ are the $x$-coordinate and the $y$-coordinate of $P_{1}$ respectively. Let $g(T)$ be the formal power series obtained from (18) by substituting $z=\ell(T)$, i.e.,

$$
\begin{aligned}
g(T)= & \alpha^{12} \Delta(L)^{1-N \alpha} \prod_{\beta}\left[-T^{-2} a(T)-p\left(\mu \tau_{2}\right)\right. \\
& \left.+\frac{1}{4}\left(\frac{-2 T^{-3} a(T)-p^{\prime}\left(\mu \tau_{2}\right)}{T^{-2} a(T)-p\left(\mu \tau_{2}\right)}\right)^{2}-p(\beta)\right]^{6} .
\end{aligned}
$$

Since $\left(f_{0}, \pi\right)=1$ and $(\alpha, \pi)=1$, we see that both $p\left(\mu \tau_{2}\right)$ and $p(\beta)$ are $p$ integral elements of $\overline{\boldsymbol{Q}}$. Moreover the leading degree of $g(T)$ is not negative because, by the assumption that $\left(f_{0}\right) \neq(1), \phi\left(z+\mu \tau_{2}\right)$ is regular at $z=0$. Hence $g(T) \in \mathcal{O}_{\mathrm{q}}[[T]]$. Since $g\left(t\left(P_{1}\right)\right)=\phi\left(\tau_{1}+\mu \tau_{2}\right)$ by $(18)$, we have $f(\Lambda)=$ $\phi\left(\tau_{1}+\mu \tau_{2}\right)$ for the power series $f(T)=g\left(w^{-1}(T)\right) \in \mathcal{O}_{q}[[T]]$. Note that the constant term of $f(T)$ is equal to $\alpha^{12} \Delta^{1-N \alpha} \prod_{\beta}\left(p\left(\mu \tau_{2}\right)-p(\beta)\right)^{6}$, which is a unit of $\mathcal{O}_{q}$. Then we have the expansion

$$
T \frac{f^{\prime}(T)}{f(T)}=\sum_{k=0}^{\infty} b_{k} T^{k},
$$


with $b_{k} \in \mathcal{O}_{a}$. From the definition of $\psi_{k}$, it is seen that

$$
\psi_{k}\left(\phi\left(\tau_{1}+\mu \tau_{2}\right)\right)=b_{k} \bmod \mathfrak{m}_{\mathrm{q}}
$$

for $1 \leqq k<q-1$. As is well-known (see, for example, Lemma 7 of [2] or Lemma 44 of [11]), we have, for the isomorphism $\ell \circ w^{-1}$ from $\mathscr{E}$ to $G_{a}$,

$$
\ell \circ w^{-1}(T) \equiv T \bmod \text { degree } q .
$$

Hence

$$
f(T) \equiv g\left(\ell^{-1}(T)\right) \bmod \text { degree } q .
$$

Here the right hand side is equal to the power series obtained from (18) by replacing $z$ by $T$. Therefore,

$$
b_{k}=12\left[\alpha^{k} d_{k}(\alpha \mu)-(N \alpha) d_{k}(\mu)\right] \quad \text { if } k<q .
$$

This completes the proof.

Proof of the theorem. The congruences (16) will be obtained by applying $\psi_{(q-1) / 3}$ and $\psi_{2(q-1) / 3}$ to both sides of (7). We only consider the first congruence because the second one can be proved similarly. Put $k=(q-1) / 3$. Let $\gamma, \beta_{j} \in \mathcal{O}_{K}$ and $m_{j} \in Z(j \in J)$ be the integers fixed in Section 2. We first calculate $\psi_{k}\left(N_{H_{f} / H}(\phi(\tau ; \gamma))\right)$. Set $\phi(z)=\phi(z ; \gamma)$. By Lemma 2, we have

$$
\phi(\tau)^{\sigma_{\alpha}}=\phi\left(\alpha^{*} \tau\right)=\phi(\alpha \tau)
$$

for any $\alpha \in \mathcal{O}_{K},(\alpha, 6 f)=1$. Here $\sigma_{a}$ is the Artin automorphism of $(\alpha)$ with respect to $M / K$. Since $\mathrm{Cl}(f)$ is isomorphic to $\left(\mathcal{O}_{K} / f \mathcal{O}_{K}\right)^{\times} / \bar{\mu}$ and the number of elements of $\bar{\mu}$ is 6 , we see

$$
\begin{aligned}
N_{H_{f} / H}(\phi(\tau))^{18} & =\prod_{\substack{\alpha \bmod f \\
x(\alpha)=1}} \phi(\alpha \tau)^{3} \\
& =\prod_{\substack{\alpha \bmod f \\
(\alpha, f)=1}} \phi(\alpha \tau)^{1+\chi(\alpha)+\chi^{-1}(\alpha)} \\
& =\left(N_{H_{f} / K}(\phi(\tau))\right)^{6} \prod_{\alpha \bmod f} \phi(\alpha \tau)^{x(\alpha)+\chi^{-1(\alpha)}} .
\end{aligned}
$$

Because $\psi_{k}(u)=0$ for $u \in M_{0, q}$, we obtain

$$
\begin{aligned}
& 18 \psi_{k}\left(N_{H_{f} / H}(\phi(\tau))\right) \\
& \quad=\sum_{\alpha \bmod f}\left(\chi(\alpha)+\chi^{-1}(\alpha)\right) \psi_{k}(\phi(\alpha \tau)) \\
& \quad=\sum_{\mu \bmod f_{0} \nu \bmod \pi}\left(\chi\left(\mu+\nu f_{0}\right)+\chi^{-1}\left(\mu+\nu f_{0}\right)\right) \psi_{k}\left(\phi\left(\left(\mu+\nu f_{0}\right)\left(\tau_{1}+\tau_{2}\right)\right)\right)
\end{aligned}
$$




$$
\begin{aligned}
& =\sum_{\mu} \sum_{\nu}\left(\chi_{1}\left(\mu+\nu f_{0}\right) \chi_{2}(\mu)+\chi_{1}^{-1}\left(\mu+\nu f_{0}\right) \chi_{2}^{-1}(\mu)\right) \psi_{k}\left(\phi_{k}\left(\left(\mu+\nu f_{0}\right) \tau_{1}+\mu \tau_{2}\right)\right) \\
& =\sum_{\mu} \sum_{\nu}\left(\chi_{1}(\nu) \chi_{2}(\mu)+\chi_{1}^{-1}(\nu) \chi_{2}^{-1}(\mu)\right) \psi_{k}\left(\phi\left(\nu \tau_{1}+\mu \tau_{2}\right)\right) .
\end{aligned}
$$

By Lemma 4 and by the fact that $\chi_{1}(\nu)=(\nu / \pi)_{3} \equiv \nu^{k} \bmod p$, we have

$$
\begin{aligned}
\psi_{k}\left(\phi\left(\nu \tau_{1}+\mu \tau_{2}\right)\right) & =\psi_{k}\left(\phi\left(\tau_{1}+\mu \tau_{2}\right)^{g_{\nu}}\right) \\
& =\nu^{k} \psi_{k}\left(\phi\left(\tau_{1}+\mu \tau_{2}\right)\right) \\
& =\chi_{1}(\nu) \psi_{k}\left(\phi\left(\tau_{1}+\mu \tau_{2}\right)\right)
\end{aligned}
$$

if $\nu$ is prinie to $\pi$. Hence, by the Proposition,

$$
\begin{aligned}
18 \psi_{k}\left(N_{H_{f} / H}(\phi(\tau))\right) & =\sum_{\mu} \sum_{\substack{\nu \bmod \pi \\
(\nu, \pi)=1}}\left(\chi_{1}(\nu)^{2} \chi_{2}(\mu)+\chi_{2}^{-1}(\mu)\right) \psi_{k}\left(\phi\left(\tau_{1}+\mu \tau_{2}\right)\right) \\
& =(q-1) \sum_{\mu} \chi_{2}^{-1}(\mu) \psi_{k}\left(\dot{\phi}^{\prime}\left(\tau_{2}+\mu \tau_{2}\right)\right) \\
& =12\left(\gamma^{k} \chi_{2}(\gamma)-N \gamma\right) G_{k, \chi_{2}} \bmod \mathfrak{m}_{\mathrm{q}} \\
& =12(\chi(\gamma)-N \gamma) G_{k, \chi_{2}} \bmod \mathfrak{m}_{\mathrm{q}} .
\end{aligned}
$$

Similar formulas hold for $\phi\left(\tau ; \gamma^{\prime}\right), \phi\left(\tau ; \beta_{j}\right)$ and $\phi\left(\tau ; \beta_{j}^{\prime}\right)(j \in J)$, and we get

$$
\begin{aligned}
& \psi_{k}\left(N_{H H_{j} / H}(\eta \bar{\eta})\right) \\
& \quad=\frac{2}{3}\left[\chi(\gamma)+\chi\left(\gamma^{\prime}\right)-2 N \gamma+\sum_{j \in J} m_{j}\left(\chi\left(\beta_{j}\right)+\chi\left(\beta_{j}^{\prime}\right)-2 N \beta_{j}\right)\right] G_{k, \chi_{2}} \bmod \mathfrak{m}_{\mathrm{a}} .
\end{aligned}
$$

Note that $\chi\left(\alpha^{\prime}\right)=\chi^{-1}(\alpha)$ for any $\alpha \in \mathcal{O}_{K}$. Then it follows from (5) that the number in the square bracket is equal to

$$
-1-2 N \gamma+2 \sum_{j \in J} m_{j}\left(1-N \beta_{j}\right)=-3 .
$$

This gives

$$
\psi_{k}\left(N_{H_{f} / H}(\eta \bar{\eta})\right)=-2 G_{k, \chi_{2}} \bmod \mathfrak{m}_{\mathrm{q}} .
$$

On the other hand, we have, by the definition of $\sqrt[3]{m_{0}}$,

$$
\frac{\varepsilon}{t}=1-\frac{u}{t} \sqrt[3]{m_{0}} \Lambda^{k}+\frac{v}{t} \sqrt[3]{m_{0}^{2}} \Lambda^{2 k}
$$

Therefore,

$$
\psi_{k}(\varepsilon)=\psi_{k}\left(\frac{\varepsilon}{t}\right)=\frac{u}{3 t} \sqrt[3]{m_{0}} \bmod m_{a}
$$

Hence, by (7), 


$$
-2 \frac{u}{t} h \equiv G_{k, \chi_{2}} / \sqrt[3]{m_{0}} \bmod \mathfrak{m}_{\mathrm{q}}
$$

We complete the proof of the first congruence of (16) by observing that the both sides of the above congruence are contained in $K$.

Finally, suppose $p \equiv-1 \bmod 3$. Then $N_{Q(\delta) / Q}(\varepsilon)=1$ gives $t^{3} \equiv 1 \bmod p$, hence $t \equiv 1 \bmod p$.

ExAmple. Take $m=10, p=5$. Then $h=1, \varepsilon=\left(23+11 \sqrt[3]{10}+5 \sqrt[3]{10^{2}}\right) / 3$ (Wada [13]), and

$$
-2 u h \equiv 1, \quad 2\left(2 v-u^{2}\right) h \equiv 2 \bmod 5 .
$$

On the other hand, we see $f=10, f_{0}=-2$, and

$$
\left(\mathcal{O}_{K} / f_{0} \mathcal{O}_{K}\right)^{\times}=\left\{\bar{\zeta} \mid \zeta^{3}=1\right\},
$$

where the bar denotes the residue class modulo $f_{0}$. By (10),

$$
\chi_{2}(\zeta)=\left(\frac{\zeta}{5}\right)_{3}^{-1}=\zeta \quad \text { if } \zeta^{3}=1
$$

Furthermore, the equations $4 p\left(\tau_{2}\right)^{3}-1=p^{\prime}\left(\tau_{2}\right)^{2}=0\left(\tau_{2}=\Omega / 2\right)$ give

$$
p\left(\zeta \tau_{2}\right)=\zeta p\left(\tau_{2}\right)=\zeta \sqrt[3]{4}^{-1} \quad \text { if } \zeta^{3}=1 .
$$

Hence, we see from (12)

$$
\frac{1}{2} \sum_{\zeta^{3}=1} \zeta^{-1} \frac{p^{\prime}(z)}{p(z)-\zeta \sqrt[3]{4}^{-1}}=-\sum_{k=1}^{\infty} G_{k, x_{2}} z^{k-1}
$$

Similar formula holds for $G_{k, x_{2}^{-1}}$. The differentiation of $p^{\prime}(z)^{2}=4 p(z)^{3}-1$ gives $p^{\prime \prime}(z)=6 p(z)^{2}$, from which follows

$$
p(z)=\frac{1}{z^{2}}+\frac{1}{28} z^{4}+\frac{1}{10192} z^{10}+\cdots .
$$

Thus we obtain

$$
G_{8, \chi_{2}}=\frac{3^{2} \sqrt[3]{2}}{2^{3} 7}, \quad G_{16, \chi_{2}}=\frac{3^{2} 19 \sqrt[3]{2^{2}}}{2^{6} 7^{2} 13}
$$

Since $\sqrt[3]{m_{0}}=-\sqrt[3]{2}$

$$
G_{8, x_{2}} / \sqrt[3]{m_{0}}=-\frac{3^{2}}{2^{3} 7} \equiv 1 \quad \bmod 5
$$




$$
G_{16, x_{2}^{-1}} / \sqrt[3]{m_{0}^{2}}=\frac{3^{2} 19}{2^{6} 7^{2} 13} \equiv 2 \quad \bmod 5
$$

and we see the congruence (16) hold.

Remark. Let $K_{4}$ be a real pure quartic field and $K_{2}$ the quadratic subfield of $K_{4}$. Let $H_{+}$be the group of positive relative units of $K_{4} / K_{2}$, and $\varepsilon_{0}(>1)$ the generator of $H_{+}$, i.e.,

$$
H_{+}=\left\{\varepsilon \in E \mid \varepsilon>0, N_{K_{4} / K_{2}}(\varepsilon)=1\right\}=\left\langle\varepsilon_{0}\right\rangle .
$$

Here $E$ denotes the group of all units of $K_{4}$. Then, we can formulate a class number formula such as

$$
\varepsilon_{0}^{h_{4} / h_{2}}=\text { (the elliptic unit), }
$$

where $h_{4}$ and $h_{2}$ denote the class number of $K_{4}$ and that of $K_{2}$ respectively (cf. Nakamula [9] and the papers quoted there). Taking Kummer's logarithmic derivatives of both sides, we will be able to obtain congruence relations similar to (16). ${ }^{2)}$ The same procedure will apply to pure sextic fields.

\section{§6. $P$-adic $L$-functions}

In the special case that $p$ splits in $K$, we can also derive our congruence relations (16) from the discussion concerning the $p$-adic $L$-functions associated with the elliptic curve $E$. Throughout this section, we assume $p \equiv 1 \bmod 3$. Recall that the algebraic numbers in $C_{p}$ are identified with those in $C$ via $i_{\infty}$ and $i_{p}$. We shall work mainly in $C_{p}$.

Let $\mathscr{T}=(E, d x / 2 y, r)$ be a triple consisting of our elliptic curve $E$, the invariant differential $d x / 2 y$ on $E$, and an isomorphism $r$ of formal groups from the formal multiplicative group $G_{m}$ (i.e., $G_{m}(X, Y)=X+Y$ $+X Y)$ to $\hat{E}$, with coefficients in $\mathcal{O}\left(K_{\mathfrak{p}, n r}^{\wedge}\right)$. Here $K_{\mathfrak{p}, n r}^{\wedge}$ denotes the completion of the maximal unramified extension of $K_{p}$. The existence of $r$ follows from Lemma 2 of [7]. Further, put $\chi=(\mathrm{m} / \cdot)_{3}$ and let $P$ be the $f$-division point on $E$ fixed in Section 3. With these data, Lichtenbaum [6] associated $C_{p}$-valued continuous functions $L(\mathscr{T}, \chi, P)(s)$ and $L\left(\mathscr{T}, \chi^{-1}, P\right)$. (s) on $Z_{p}$. Take a positive integer $N$ such that $\chi(N) \neq 0,1$. Then by Theorem 8.11 of [6] any by the definition of $L(\mathscr{T}, \chi, P)$, we can write

$$
L(\mathscr{T}, \chi, P)(s)=h\left((1+p)^{s}-1\right) /\left(\chi(N)\langle N\rangle^{-s+1}-1\right)
$$

2) These congruence relations have been obtained by Masato Kamei. 
for some $h(T) \in \mathcal{O}\left(C_{p}\right)[[T]]$. Here, $\langle N\rangle$ is a $p$-adic integer determined by

$$
\begin{aligned}
& N=\omega(N)\langle N\rangle, \\
& \omega(N)^{p-1}=1, \quad \omega(N) \equiv N \bmod p .
\end{aligned}
$$

Hence, we have

$$
L(\mathscr{T}, \chi, P)(m) \equiv L(\mathscr{T}, \chi, P)(n) \quad \bmod p
$$

for any rational integers $m$ and $n$.

Now, taking the $p$-adic logarithms of (3), we obtain

$$
12 f h \log _{p} \varepsilon=-\frac{1}{6} \sum_{\substack{\alpha \bmod f \\(\alpha, f)=1}}\left(\chi(\alpha)+\chi^{-1}(\alpha)\right) \log _{p} \varphi_{f}\left(C_{\alpha}\right) .
$$

Define a primitive $p$-th root of unity $\zeta$ by $\zeta-1=r^{-1}\left(t\left(P_{1}\right)\right)$, and put

$$
S_{\chi}=\tau\left(\chi_{1}, \zeta\right) / p, \quad \tau\left(\chi_{1}, \zeta\right)=\sum_{a=1}^{p-1} \chi_{1}(a) \zeta^{a} .
$$

By Corollary 4.2 of [6], we can define a unit $u_{0}$ of $K_{p, n r}^{\wedge}$ by

$$
\ell^{-1}(T)=r\left(e^{u_{0} T}-1\right) .
$$

Then, if we put $k=(p-1) / 3$, it follows from Corollary 9.4 of [6] (Note that the left hand side of the formula of Corollary 9.2 and the right hand side of the formula of Corollary 9.4 should be multiplied by $1 / 2$ ), the formula (20), and Theorem 8.2 of [6], that

$$
\begin{aligned}
\frac{1}{6 f} \sum_{\substack{\alpha \bmod f \\
(\alpha, f)=1}} \chi^{-1}(\alpha) \log _{p} \varphi_{f}\left(C_{\alpha}\right) & =2 S_{\chi}^{-1} u_{0}^{-1} L(\mathscr{T}, \chi, P)(1) \\
& \equiv 2 S_{\chi}^{-1} u_{0}^{-1} L(\mathscr{T}, \chi, P)(1-k) \\
& \equiv 6 k ! S_{\chi}^{-1} u_{0}^{-k} G_{k, \chi_{2}} \bmod p .
\end{aligned}
$$

Lemma 5. (i) $S_{\chi}^{-1} \equiv-\pi^{\prime}(k !)^{-1} u_{0}^{-2 k} \Lambda^{k} \bmod p$.

(ii) $u_{0}^{p-1} \equiv \pi^{\prime} \bmod p$.

The proof will be given later. By this lemma, we get

$$
\frac{1}{6 f} \sum_{\alpha} \chi^{-1}(\alpha) \log _{p} \varphi_{f}\left(C_{\alpha}\right) \equiv-6 G_{k, x_{2}} \Lambda^{k} \bmod p
$$

Similar consideration gives

$$
\frac{1}{6 f} \sum_{\alpha} \chi(\alpha) \log _{p} \varphi_{f}\left(C_{\alpha}\right) \equiv-3 G_{2 k, \chi_{\mathbf{\Omega}}^{-2}} \Lambda^{2 k} \bmod p .
$$

On the other hand, we see, from $t^{3} \equiv 1 \bmod p$ and (19), 


$$
\log _{p} \varepsilon \equiv \log _{p}\left(\frac{\varepsilon}{t}\right) \equiv-\frac{u}{t} \sqrt[3]{m_{0}} \Lambda^{k}+\frac{1}{2}\left(2 \frac{v}{t}-\left(\frac{u}{t}\right)^{2}\right) \sqrt[3]{m_{0}{ }^{2}} \Lambda^{2 k} \bmod p
$$

Then we obtain the congruences (16) from (21).

Proof of Lemma 5. By (22),

$$
r(T) \equiv u_{0}^{-1} T \bmod \text { degree } 2,
$$

hence

$$
\zeta-1 \equiv u_{0} t\left(P_{1}\right) \equiv u_{0} \Lambda \bmod \Lambda^{2}
$$

On the other hand, as is well-known (e.g., see Weil [14]), we have

$$
\begin{aligned}
& \tau\left(\chi_{1}, \zeta\right) \equiv k !(\zeta-1)^{2 k} \bmod \Lambda^{2 k+1}, \\
& \tau\left(\chi_{1}, \zeta\right)^{3}=-(-1)^{(p-1) / 2} p \pi \\
& \left(\frac{\pi^{\prime}}{\pi}\right)_{3}=1 .
\end{aligned}
$$

It follows from (14) that

$$
\tau\left(\chi_{1}, \zeta\right) / \Lambda^{2 k} \in K_{p}\left(\sqrt[3]{\pi^{\prime}}\right)=K_{p} .
$$

Then, by (23) and (24),

$$
\tau\left(\chi_{1}, \zeta\right) / \Lambda^{2 k} \equiv k ! u_{0}^{2 k} \bmod p .
$$

Therefore we obtain

$$
S_{\chi}^{-1}=-\pi^{\prime} \Lambda^{3 k} / \tau\left(\chi_{1} \cdot \zeta\right) \equiv-\pi^{\prime}(k !)^{-1} u_{0}^{-2 k} \Lambda^{k} \bmod p .
$$

To prove (ii), observe that the isomorphism $r$ from $G_{n}$ to $\hat{E}$ satisfies

$$
r\left([p]_{G_{m}}(T)\right)=[\pi]_{\hat{E}}\left(r\left(\left[\pi^{\prime}\right]_{G_{m}}(T)\right)\right) .
$$

Comparing the coefficients of $T^{p}$, we obtain (ii).

Acknowledgement. I would like to thank Masao Koike, Hiromichi Yanai, and Hideo Yokoi for helpful suggestions on the manuscript.

\section{REFERENCES}

[1] N. Ankeny, E. Artin and S. Chowla, The class number of real quadratic fields, Ann. of Math., (2), 56 (1952), 479-493.

[2] J. Coates and A. Wiles, On the conjecture of Birch and Swinnerton-Dyer, Invent. math., 39 (1977), 223-251.

[3] H. Davenport and H. Hasse, Die Nullstellen der Kongruenz-zetafunktionen in gewissen zyklischen Fällen, J. reine angew. Math., 172 (1935), 151-182. 
[4] H. Hasse, Arithmetische Theorie der kubischen Zahlkörper auf klassenkörpertheoretischer Grandlage, Math. Z., 31 (1930), 565-582.

[ 5 ] W. J. LeVeque, Topics in number theory, Vol. II, Reading, Mass., 1961.

[ 6 ] S. Lichtenbaum, On $p$-adic $L$-functions associated to elliptic curves, Invent. math., $56(1980), 19-55$.

[ 7 ] J. Lubin and J. Tate, Formal complex multiplication in local fields, Ann. of Math., 8 (1965), 380-387.

[ 8 ] C. Meyer, Die Berechnung der Klassenzahl abelscher Körper uber quadratischen Zahlkörpern, Akademie-Verlag, 1957.

[ 9 ] K. Nakamula, Class number calculation and elliptic units. I, II, III, Proc. Japan Acad., 57A (1981), 56-59, 117-120, 363-366.

[10] G. Robert, Unités elliptiques, Bull. Soc. Math. France, Mém., 36 (1973).

[11] — Numbre de Hurwitz et unités elliptiques, Ann. Sci. École Norm. Sup., $4^{\mathrm{e}}$ série, 11 (1978), 297-389.

[12] J. Tate, The arithmetic on elliptic curves, Invent. math., 23 (1974), 179-206.

[13] H. Wada, A table of fundamental units of pure cubic fields, Proc. Japan Acad., 46 (1970), 1135-1140.

[14] A. Weil, La cyclotomie jadis et naguère, Sém. Bourbaki, 1973/1974, Exp. no. 452, Springer Lecture Notes in Math., Vol. 431 (1975), 318-338.

Department of Mathematics

Nagoya University

Chikusa-ku, Nagoya, 464

Japan 